\title{
A systematic approach to reduce the independent tensor components by symmetry transformations: a commented translation of "Tensors and Crystal Symmetry" by Carl Hermann
}

Received: 2 November 2020 / Accepted: 20 January 2021 / Published online: 24 March 2021

(C) The Author(s) 2021

\begin{abstract}
We here present a faithful translation of Carl Hermann's important 1934 paper "Tensoren und Kristallsymmetrie". This work, originally published in German language, is transferred into English while the preceding foreword summarizes Hermann's achievements.
\end{abstract}

Keywords Crystal symmetry · Symmetry groups · Elasticity · Fourth-order tensor · Hermann's theorem

\section{Foreword}

Introduction

The work of Carl Hermann on the representation of explicit invariance requirements on tensor components due to crystal symmetries is undoubtedly a milestone in the theory of anisotropic materials. He published his approach in an article which appeared in Zeitschrift für Kristallographie (German for Journal of Crystallography, now Zeitschrift für Kristallographie-Crystalline Materials ${ }^{1}$ ) in 1934; cf. Hermann [13]. However, this work is not well known since it was published in German language solely.

Carl Hermann (1898-1961) was a German physicist whose work focused on crystallography. He studied in Göttingen where he finished in 1923 his dissertation ${ }^{2}$ under the supervision of Max Born (1882-1970). He then moved to Stuttgart where he became an assistant of Paul Peter Ewald (1888-1985). In Stuttgart, he

\footnotetext{
1 Journal homepage http://www.degruyter.com/view/j/zkri.

2 The title of this dissertation is On the natural optical activity of some regular crystals ( $\mathrm{NaClO3}$ and NaBrO3): A proof of Born's theory of crystal optics (englisch for Über die natürliche optische Aktivität von einigen regulären Kristallen (NaClO3 und $\mathrm{NaBrO3):} \mathrm{eine} \mathrm{Prüfung} \mathrm{der} \mathrm{Bornschen} \mathrm{Theorie} \mathrm{der} \mathrm{Kristalloptik)} \mathrm{which} \mathrm{was} \mathrm{later} \mathrm{published} \mathrm{in} \mathrm{Hermann} \mathrm{[11].}$

${ }^{3}$ The title of the habilitation thesis is The symmetry groups of the amorphous and mesomorphous phases (english for Die Symmetriegruppen der amorphen und mesomorphen Phasen) which was later published in Hermann [12].
}

Communicated by Victor A. Eremeyev and Andreas Öchsner.

Supplementary Information The online version contains supplementary material available at https://doi.org/10.1007/ s00161-021-00978-5.

R. Glüge ( $\varangle)$

Faculty of Mechanical Engineering, Institute of Mechanics, Otto von Guericke University, Universitätsplatz 2,

Magdeburg 39106, Germany

E-mail: gluege@uni-bremen.de

R. Glüge · M. Aßmus

Department of Applied and Structural Mechanics, Faculty of Production Engineering, University of Bremen,

Am Biologischen Garten 2, 28359 Bremen, Germany 
had first contact with research on crystal structures, which was a topic on upswing at that time. In 1930, he finished his habilitation. ${ }^{3}$ Hermann brought new aspects to crystallography and made important contributions to classification, structure and nomenclature of space groups [20].

During the Nazi dictatorship in Germany between 1933 and 1945, Hermann had to leave the university, as he refused to engage in political activities. Hermann worked for the IG Farben (Interessengemeinschaft Farbenindustrie $A G$ ) in Ludwigshafen am Rhein, where he edited publications on the fine structure of crystalline matter and chemical bonds with Rudolf Brill, Hans Georg Grimm and Claus Peters. His wife Eva and he were arrested and sentenced to prison for protecting fellow Jews from deportation by helping them to escape. Only his fame as a scientist and the intervention of his friends saved them from being executed. ${ }^{4} \mathrm{He}$ stayed in Germany and conducted further investigations on symmetries of crystals.

Today, the German Society for Crystallography awards the Carl Hermann Medal as its highest honor. ${ }^{5}$

\section{Discussion}

In the middle of the nineteenth century, it became clear that 32 crystal classes can be distinguished, which result from the crystallographic restriction theorem, which states that only two-, three-, fourfold and sixfold rotational symmetries are possible in crystals. It was found independently by various researchers, namely Auguste Bravais, Johann Friedrich Christian Hessel, Moritz Ludwig Frankenheim and Axel Wilhelmowitsch Gadolin [16], but mostly Voigt [23] is cited. Later quasiperiodic crystals [2] with other than the above mentioned symmetries have been found. They are not periodic in a strict sense; hence, no point groups are associated, but still they exhibit an aperiodic order, which one might call an effective symmetry.

The crystal classes are usually summarized in 7 crystal families. The unifying feature in each family is a common rotational symmetry:

- The triclinic family has no rotational symmetries,

- the monoclinic family has one twofold rotation axis $\mathbb{Z}_{2}$,

- the orthotropic (orthorhombic) family has three orthogonal twofold rotation axis that can be generated by two orthogonal twofold rotations $\mathbb{Z}_{2} \times \mathbb{Z}_{2}$,

- the trigonal family has one threefold rotation axis $\mathbb{Z}_{3}$,

- the tetragonal family has one fourfold rotation axis $\mathbb{Z}_{4}$,

- the hexagonal family has one sixfold rotation axis $\mathbb{Z}_{6}$,

- the cubic symmetry has three orthogonal fourfold rotations axes.

The differences within each family are additional symmetries which may be present, e.g. additional twofold rotation axes or a centro-symmetry. The relation between the crystal classes and the subgroups of the orthogonal group $O$ (3) with $\operatorname{det} \mathbf{Q}= \pm 1$ for $\mathbf{Q} \in O$ (3) is by no means trivial. Luckily, in classical elasticity only symmetry groups which are subgroups of the special orthogonal group $\mathrm{SO}(3)$ with $\operatorname{det} \mathbf{Q}=1$ for $\mathbf{Q} \in \mathrm{SO}$ (3) need to be distinguished. This is due to the fact that any element of the orthogonal group combines with $-\mathbf{I}$, which is in $O(3)$, to an element of $\mathrm{SO}(3)$, which is achieved by a mere change of sign of $\mathbf{Q}$. Since the stiffness tetrad is a tensor of even order, this change of sign cancels out, which is why it is sufficient to only examine $\mathbf{Q}$ with positive determinant, i.e. elements of $\mathrm{SO}(3)$. Traditionally, the deduction of restrictions on the stiffness tetrad by material symmetries is done applying invariance of the stiffness under the rotation by generators of a specific symmetry group. This leads to restrictions for specific symmetry groups, but an overarching approach for the whole symmetry class is not obtained. The latter was put forward by Forte and Vianello [9], who used the harmonic decomposition and rigorously showed that 8 symmetry classes can be distinguished in elasticity. A symmetry class is the abstract set of all symmetry groups which are isomorphic to each other. Hermanns work is an elegant execution of the more accessible but less general group-specific approach, which is why he found 9 distinguishable symmetry classes. It was found later by ХаТКеВИЧ [25] that two of them differ by a simple rotation; hence, they fall into the same class. A systematic and rigorous proof of the number of distinguishable symmetry classes requires more abstract approaches like the harmonic decomposition [9] ot the irreducible decomposition [26].

The more accessible group-specific approach and the more abstract symmetry-class-specific approaches lead to different numbers of independent components in the stiffness tetrad because the orientation parameters

\footnotetext{
${ }^{4}$ Fame as a scientist was no protection from being killed by the Nazis, as the examples of Felix Hausdorff and many others shows.

${ }^{5}$ List of laureates: https://dgk-home.de/intern/ehrenmitglieder-und-preistraeger/preistraeger-der-carl-hermann-medaille.
} 
are often fixed in the first case while they do not appear in the second case. For example, for cubic symmetry usually 3 independent components of the stiffness tetrad are given while for a triclinic material 21 independent components are declared. Clearly, the 21 moduli contain the orientation, unlike the three elastic moduli in the cubic case. Also, the number of orientation parameters may be zero (isotropy), two when only a single axis defines the orientation (monoclinic, transversaly isotropic) or three when the full orientation information is needed. For example, Kowalczyk-Gajewska and Ostrowska-Maciejewska [15] take into account the orientation information as unknown parameters.

Hermann has formalized and simplified the application of invariance requirements due to material symmetries for constitutive tensors of arbitrary order. He gives a methodology to formulate the linear constraints that emerge between the tensor components. It is stated apparently for the first time that the maximum count $n$ of $n$-fold cyclic symmetries that can be distinguished is the tensorial order $o$ plus one, namely the $n$-fold cyclic symmetries up to $o$, while for all $n>o$ the same invariance requirements emerge, which are these of continuous cyclic symmetry. Hermann achieves this essentially through a diagonalization of orthogonal tensors in a complex Euclidean space. He presents the technique and exemplifies it by deriving the 10 classical symmetry groups and the corresponding linear constraints on the tensor components that can be distinguished in linear elasticity on only 4 pages.

In more recent publications, e.g. in Nordmann et al. [19], only 8 symmetry classes are distinguished. The difference to ten comes from two cyclic trigonal and tetragonal groups $C 3$ and $C 4$, which are simple threefold and fourfold rotational symmetries around an axis we call $c$. Both leave seven different, independent components in the stiffness tetrad. If one adds a twofold rotation around an axis perpendicular to $c$, one obtains the dihedrel trigonal and tetragonal groups $D 3$ and $D 4$, which leave six independent components in the stiffness tetrad.

It has later been found by ХаТКеВИЧ [25] (or also Khatkevich [14]) that the reduced stiffness tetrads due to $C 3, D 3$ and $C 4, D 4$ differ only by a rotation around the threefold or fourfold rotation axis, which allows to express the seven independent components in case of the cyclic groups $C 3$ and $C 4$ through the 6 independent components from the dihedral groups $D 3$ and $D 4$ plus the rotation angle. The rotation angle depends in a complicated way on the actual components. The same result has been obtained by Fedorov [8], Cowin [4], Ting [21], Forte and Vianello [9]. Gurtin [10] still lists ten different matrices, which contain the above-mentioned redundancies.

Equally important, the clear connection between the symmetry classes that can be distinguished and the tensorial order reminds us of the fact that linearity, as desirable a property it may be, does not only confine us to a small interval of the constitutive law where linearity is deemed a good approximation: it also limits the symmetries that can be distinguished. There are examples for anisotropic heat conduction in cubic crystals [17] —admittedly at extreme temperatures — where a clear cubic anisotropy of the conductivity is measured. But since the tensor of conductivity is of second order, isotropy and cubic symmetry cannot be distinguished. This may well occur in other contexts, but it is rarely tested whether such an indistinguishablity is acceptable.

\section{Conclusion}

Hermann demonstrated a derivation of distinct material symmetries for the elasticity tensor by employing an, at least at this time, ingenious technique which is based on a diagonalization of orthogonal tensors with respect to complex axes.

Unfortunately, important results like obtained by Hermann [13] are forgotten or ignored, and later rediscovered. Usually this happens in a different context or discipline, so that an interdisciplinary expertise is required. Then, however, errors usually still occur due to poor translations, since the scientific language has changed in the meantime. With the present translation, we hope to contribute to a dissemination of the knowledge of the work by Hermann which up to now was mostly restricted to German speaking scientists. Even if almost 90 years have passed since the publication of the original work, the topic still seems to be of interest (cf. Chadwick et al. [3]) whereby it gains relevance especially in the context of generalized continuum theories, e.g. Pietraszkiewicz [6] or Auffray et al. [1].

The reading of the original article is hard due to the partial use Fraktur font and a somewhat cumbersome linguistic style. Therefore we have updated the notation and linguistic style to one that is common today. A table in "Appendix A" provides the relations of original and updated notation and explains their purpose. The equation numbering of Hermann's original paper has been maintained. However, the numbering of footnotes 
has been amended due to page breaks and additional remarks of the translators. A source code written in Mathematica ${ }^{\circledR}$ given in "Appendix B" reconstructs Hermann's results computationally.

Hermann uses upper- and lowercase letters for the indices as well as for the tensor component. For indices, the case distinction is simply used to have more indices available, such that one can write a transformation rule like $C_{i j k l}=Q_{i j k l I J K L} C_{I J K L}$. The uppercase indices are in this example summation indices for which other symbols could have been used, but the case distinction distinguishes them from the free indices. For the tensor symbol, the case distinction implies that a component is represented in all indices w.r.t. the complex basis (upper case) or the real basis (lower case). The position as super- or subscript indicates the co- or contravariant character of the index. Polar and axial tensors are not distinguished typographically.

\section{Tensors and Crystal Symmetry by C. Hermann in Stuttgart}

\section{Zeitschrift für Kristallographie, Band 89, Heft 1, 32-48, 1934}

A method for deriving the effect of the crystal symmetry on the material constants of arbitrary tensorial order is given (vanishing components, number of linearly independent components, linear constraints between components). This is done by choosing appropriate—in general complex-coordinate axes.

\section{Aim of this work}

The following statements refer to a very large number of groups of material constants, namely all those that define a proportionality between an arbitrary action on matter and its reaction. Such constants are, for example, the specific heat $c_{p}\left(Q=c_{p} \cdot \Delta \theta\right)$, the dielectric constant $\varepsilon(D=\varepsilon E)$, the electrical conductivity $\sigma(j=\sigma E)$, the thermal expansion coefficient $\alpha(\Delta l / l=\alpha \Delta T)$ or the elastic compressibility $\kappa(\Delta V / V=\kappa \Delta p)$. In isotropic matter, such constants are in general ordinary (scalar) numbers and invariant to the choice (orientation) of the coordinate axes. In anisotropic matter, however, in crystals, smectic or nematic bodies or in glasses in anisotropic states of stress, they become orientation dependent in various ways. Among the mentioned examples only the specific heat, being a »scalar «, is invariant to coordinate transformations, while $\varepsilon, \sigma$ and $\alpha$ behave like second order tensors and even $\kappa$ like a fourth order tensor.

It may happen that there is no longer exact proportionality between action and reaction, that for example the specific heat becomes dependent on the temperature, or that the electrical polarization of matter cannot exceed a saturation value. Then it is often still possible to write the constitutive law in the form of a power series, where higher tensorial series coefficients of generally different order appear. Only when such a description no longer is sufficient, as in the case of splitting or sliding processes, the description of a law of nature by tensorial material constants is insufficient.

It is known that the individual material constant generally only allow a distinction to be made between certain groups of crystal classes, but is subject to exactly the same conditions for the group members. Among the properties mentioned, the specific heat does not allow to distinguish any classes of symmetry from each other. The dielectric constant (as well as the optical birefringence to be derived from it) and the thermal expansion allow the differentiation of 5 groups: the triclinic, monoclinic, rhombic, uniaxial and regular group, the uniaxial group comprising all crystal classes with a single axis of rotation or rotation-reflection axis of count 3,4 or 6 . The conductivity, which is a second-order tensor, as well as the two mentioned properties, also allows to distinguish among the uniaxial classes those with horizontal minor axes or vertical reflection planes from the others. Thus, each tensor material property divides the crystal classes into a number of distinguishable groups, which generally still contain a number of different classes distinguished by crystal classes.

The following investigation is intended to provide an overview of this type of classification by properties described by tensors of any order and also to derive the relationships between the tensor components required by the symmetry elements of the crystal.

The results of the work will in most individual cases be nothing new. In his book, Voigt ${ }^{6}$ has calculated such formulas for numerous cases. His idea is to derive scalar functions from the tensor components, mostly an energy or a power, and for these scalars he requires invariance with respect to the symmetry operations of the crystal. One difficulty in his considerations is that in each case a suitable scalar must be sought anew and that one is then usually not yet sure whether the invariance of this scalar also brings with it all invariance

\footnotetext{
${ }^{6}$ Voigt, W., Lehrbuch der Krystallphysik, Leipzig, 1928; see Voigt [23].
} 
properties that one must naturally demand for the whole tensor. In the following we will therefore not only look for the invariance condition for such a scalar but for each tensor component in detail, and we will secondly use a calculus that remains independent of the tensor's order as long as possible, and only at the end introduce the tensorial order where it is easily kept track of. In this way, we get a number of theorems about the distinguishability of crystal classes by tensors of different order.

\section{Notation}

The notation used here for the tensor calculus can be found for example in the book by $\mathrm{H}$. W e y $\mathrm{l}^{7}$. A tensor of $n$th order in three-dimensional space can be decomposed into $3^{n}$ components according to any $3 \mathrm{D}$ vector basis $a_{1}, a_{2}, a_{3}$. One writes the components by a basic letter for the tensor and $n$ indices, which take the values 1,2 , or 3 independently. A distinction must be made between covariant and contravariant indices: going from one vector triple $a_{i}$ to another triple $a_{i}^{\prime}$ by the transformation

$$
a_{i}^{\prime}=\sum_{k} \beta_{i}^{k} a_{k}
$$

or its inverse

$$
a_{i}=\sum_{k} \gamma_{i}^{k} a_{k}^{\prime}
$$

the components transform linearly. For every index $i$, a factor $\beta_{i}^{k}$ or $\gamma_{i}^{k}$ appears in the transformation rule. Depending on which case we have, the tensor is covariant or contravariant. ${ }^{8}$ The simplest example is the contravariant components of the usual notation of a location vector,

$$
r=\sum_{i} x^{i} a_{i}=\sum_{i} x^{\prime i} a_{i}^{\prime}
$$

because here it holds

$$
x^{\prime i}=\sum_{k} \gamma_{k}^{i} x^{k}
$$

Contrary, the Mille r indices of a plane represent covariant components, because

$$
h^{\prime}{ }_{i}=\sum_{k} \beta_{i}^{k} h_{k}
$$

holds. A second-order tensor that is covariant in both indices is the so-called metric $g_{i k}$, which allows for determining the length of a vector $x$ from its contravariant components:

$$
|x|^{2}=\sum_{i k} g_{i k} x^{i} x^{k}
$$

Correspondingly, a second-order tensor which in both indices is contravariant gives for three M ille r indices $h_{i}$ the lattice plane distance $d$ :

$$
\frac{1}{d^{2}}=\sum_{i k} g^{i k} h_{i} h_{k}
$$

\footnotetext{
7 Weyl, H., Raum, Zeit und Materie. 5. Aufl. Berlin 1923. S. 30ff.; see Weyl [24]

8 The denominations »covariant « and »contravariant« are relative to each other: two quantities can be transformed co- or contravariant with respect to each other. When the expressions are used absolutely as in this work, a reference choice is implied, from which one decides which variables are co- and contravariant. We use, as Weyl did, the base vectors. In the literature, one finds occasionally the contrary definition, which is obtained by defining the point coordinates as the reference choice.
} 
Tensors of second order that appear as physical material constants are mostly covariant in one index and contravariant in the other. For example, for the dielectric constant

$$
D^{i}=\sum_{k} \varepsilon_{k}^{i} E^{k}
$$

(since $E^{i}$ and $D^{i}$ are both transformed contravariantly, since they are vector components related to the axes $\left.a_{i}\right)$.

If the axis transformation (1) is executed, one obtains for these tensors:

$$
g_{i k}^{\prime}=\sum_{m n} g_{m n} \beta_{i}^{m} \beta_{k}^{n} ; \quad g^{\prime i k}=\sum_{m n} g^{m n} \gamma_{m}^{i} \gamma_{n}^{k} ; \quad \varepsilon_{k}^{\prime i} \sum_{m n}=\varepsilon_{n}^{m} \gamma_{m}^{i} \beta_{k}^{n}
$$

In a similar way, covariant and contravariant indices need to be distinguished for higher-order tensors. This difference is expressed by adding covariant indices at the bottom of the symbol and contravariant indices at the top.

If one multiplies two tensors of $m$ th and $n$th order with each other in such a way that every component of one tensor is combined with each of the other, one will get $3^{m+n}$ products, which together form the components of a tensor of $(m+n)$ th order. More often than this multiplication, however, there is another one, where only such products for which at least an upper index of one tensor is identical with a lower one of the other, and where afterwards it is summed over this common index. With only one summation index this results in a tensor of $(m+n-2)$ th order. If there are more summation indices, the new tensor has the order $(m+n-4)$, $(m+n-6)$, etc. Examples are the transformation Eqs. (4), (5), (9), or the definition of the dielectric constant (8). This is the so-called c o n traction of two tensors. The reason why the order of the tensor is reduced is that during the transition from axes $a_{i}$ to $a_{i}^{\prime}$ for one index the factor $\beta_{i}^{j}$ appears and for the other the factor $\gamma_{j}^{k}$ is incorporated into the product, which is then summed over $j$. But now by inserting (2) in (1) and comparing the vector components, one obtains

$$
\sum_{j} \beta_{i}^{j} \gamma_{j}^{k}=\delta_{i}^{k}=\left\{\begin{array}{ll}
1 & \text { if } i=k \\
0 & \text { if } i \neq k
\end{array} .\right.
$$

Therefore, these two transformation components disappear during the summation and the degree of the total transformation in the $\beta_{i}^{k}$ and $\gamma_{i}^{k}$ is reduced by 2 .

If in such a product all indices appear once above and once below and are summed over, a scalar quantity is obtained, which has to be invariant under every transformation of coordinates. Examples are the vector length (6) or the lattice plane distance (7).

Due to the frequency of this contraction, we omit the summation sigma if the summation index appears once at the bottom and once at the top of the term. According to this agreement, it is therefore possible to write (6) or (8)

$$
|x|^{2}=g_{i k} x^{i} x^{k} \quad\left(6^{\prime}\right) \quad D^{i}=\varepsilon_{k}^{i} E^{k}
$$

In the first case, due to this abbreviation rule, the summation symbol $\sum_{i k}$ and in the second case the summation symbol $\sum_{k}$ has to be kept in mind.

\section{Symmetry transformations of tensors}

If the transformation matrix $\beta_{i}^{k}$ represents one of the symmetry operations of the crystal, a law of nature must be exactly the same after the transformation as before, since the rotation or reflection of a crystal at one of its symmetry elements replaces every part of matter with a completely equivalent one. ${ }^{9}$ Thus subsequent relation must be valid for any tensor of $n$th order $t_{i k l \ldots}^{p q \ldots}$ in all components:

$$
t_{I K L . .}^{P Q . .}=t_{I K L . .}^{P Q . .}=t_{i k l . .}^{p q . .} \beta_{I}^{i} \beta_{K}^{k} \beta_{L}^{l} \gamma_{p}^{P} \gamma_{q}^{Q} \ldots
$$

This is a system of $3^{n}$ linear homogeneous equations for the $3^{n}$ tensor components $t_{i k l .}^{p q \ldots}$. Besides the trivial solution of all tensor components being zero, it has other solutions if the determinant disappears. This holds

\footnotetext{
9 Translator's comment: Hermann refers to the Neumann-Curie-principle; cf. Neumann [18] and Curie [5].
} 
even if for reasons of definition or an energy principle the tensor components fulfill certain symmetries, e.g. if they are invariant under certain index permutations. Thus some of the equations become equal to each other, but at the same time the number of independent unknowns decreases in the same measure, so that the numbers of equations and unknowns remain equal. If now the determinant of degree $s$ disappears, then $s$ of the quantities $t_{i k l . .}^{p q .}$ are independent of each other and can take any values. ${ }^{10}$

The remaining components are determined by $s$ (constraints ${ }^{11}$ ). The problem of the relations between the tensor components, which are required by the symmetry elements, is thus traced back to the discussion of the determinant of such system of equations, as they are presented in (11).

There is another important distinction: there are physical properties (e.g. the optical rotation capacity or a magnetic moment) which change their sign at the transition from a right- to a left-hand coordinate system. Such properties can have the character of tensors of any order. The conditional equations like (11) for such tensors arise only if $\beta_{i}^{k}$ represents a rotation of the crystal; on the other hand, they may be of the form

$$
-t_{I K L . .}^{P Q . .}=t_{i k l . .}^{p q . .} \beta_{I}^{i} \beta_{K}^{k} \beta_{L}^{l} \gamma_{p}^{P} \gamma_{q}^{Q}
$$

for reflections or rotation-reflections. Tensors for which Eq. (11) is valid for arbitrary symmetry elements are called polar. Tensors for which the sign changes simultaneously with the direction of rotation of the coordinate axes, so that for symmetry elements of second kind Eq. $\left(11^{\prime}\right)$ is valid, are called a $\mathrm{x}$ i a .

\section{Introduction of complex axes}

The discussion of the determinants of $(11)$ or $\left(11^{\prime}\right)$ is very difficult, as long as one assumes arbitrary axis systems and therefore the measures $\beta_{i}^{k}$ and $\gamma_{i}^{k}$ can take quite confusing values during the transformation. On the other hand, it would be very simple if it were possible to choose the axes in a way that $\beta_{i}^{k}$ and $\gamma_{i}^{k}$ become diagonal matrices simultaneously. This would leave only one term of the whole $n$-fold sum on the right-hand side of the equations, where all $i k l p q$ would be equal to the corresponding indices of the left-hand side $I K L P Q$. To achieve this, it is only necessary to search for such directions, which merge into themselves during the transformation $\beta_{i}^{k}$, and to choose the coordinate axes in these directions. Thus, it is necessary to find axes

$$
A_{i}=A_{i}^{k} a_{k}
$$

such that

$$
A_{i}^{\prime}=\lambda A_{i}
$$

Now

$$
A_{i}^{\prime}=A_{i}^{k} a_{k}^{\prime}=A_{i}^{l} \beta_{l}^{k} a_{k}
$$

holds, i.e.

$$
A_{i}^{l} \beta_{l}^{k} a_{k}=\lambda A_{i}^{k} a_{k}
$$

or, by comparing the coefficients of the 3 independent vectors $a_{i}$ :

$$
A_{i}^{l} \beta_{l}^{k}=\lambda A_{i}^{k} .
$$

These are 9 linear homogeneous equations for the 9 unknowns $A_{i}^{k}$. But immediately one can see that only 3 of these unknowns are coupled, namely those with the same lower index $i$, and that the system of equations is identical for the 3 possible indices $i$. Therefore one can pick any of these 3 systems of 3 equations each. The determinant has to disappear, i.e. a third-order polynomial has to be solved for $\lambda$. This equation can have three real roots or one real and two conjugated complex roots. If we are dealing with crystallographic symmetry operations $\beta_{i}^{k}$, which leads back to the initial state after a finite number of applications, then we can see from (13) that only unit roots can be solutions for the three values $\lambda$. Thus there are only two essentially

\footnotetext{
10 Translator's comment: The rank of the linear system's coefficient matrix gives the number of independent equations, which is the number of independent constraints by which the number of independent components is reduced.

11 Translator's amendment.
} 
different complex types of symmetry elements, the axis of rotation: $\lambda_{1}=\mathrm{e}^{\mathrm{i} \varphi}, \lambda_{2}=\mathrm{e}^{-\mathrm{i} \varphi}, \lambda_{3}=+1$ and the rotation-reflection axis: $\lambda_{1}=\mathrm{e}^{\mathrm{i} \varphi}, \lambda_{2}=\mathrm{e}^{-\mathrm{i} \varphi}, \lambda_{3}=-1$. Herein $\varphi$ is the angle of the rotation that must take the value $\frac{2 \pi}{n}$ for the $n$-fold rotation or rotation-reflection axis, so that after $n$ applications the axes $A_{1}$ and $A_{2}$ are returned to the initial position. There are also three real possibilities for the $\lambda_{i}$ : the twofold axis of rotation $(-1,-1,+1)$, the reflection plane $(+1,+1,-1)$, and the center of symmetry $(-1,-1,-1)$, which, with $\varphi=0$ or $\pi$, result from the complex solutions.

If one inserts the three $\lambda$-values found as the roots of the determinant of $\left(15^{\prime}\right)$, one obtains three ratios $A_{i}^{1}: A_{i}^{2}: A_{i}^{3}$, where the ratio obtained from $\lambda_{1}$ is now simply assigned to the quantities $A_{1}^{k}$, and accordingly from $\lambda_{2}$, which determines the quantities $A_{2}^{k}$ and from $\lambda_{3}$, which determines the $A_{3}^{k}$. The absolute size of $A_{i}^{k}$ can be chosen arbitrarily, for instance by the normalization $\left|A_{1}\right|^{2}=\left|A_{2}\right|^{2}=\left|A_{3}\right|^{2}=1$. Thus, one obtains a generally complex transformation tensor $A_{i}^{k}$. The inverse transformation shall be $a_{i}^{k}$, that is

$$
A_{i}=A_{i}^{k} a_{k}
$$

Then all tensor quantities are transformed to the com-

$$
a_{i}=a_{i}^{k} A_{k} .
$$

plex axes $A_{i}$, according to the transformation theorems already given (capital letters always refer to the complex, small letters to the real axes):

$$
T_{I K L . .}^{P Q . .}=t_{i k l . .}^{p q . .} A_{I}^{i} A_{K}^{k} A_{L}^{l} a_{p}^{P} a_{q}^{Q} \ldots
$$

and

$$
t_{I K L . .}^{P Q . .}=T_{i k l . .}^{p q . .} a_{I}^{i} a_{K}^{k} a_{L}^{l} A_{p}^{P} A_{q}^{Q} \ldots .
$$

With these equations, it is possible to return at any time from the convenient but non-ostensible complex axes $A_{i}$ to the descriptive axes $a_{i}$.

The matrices $\beta_{i}^{k}$ and $\gamma_{i}^{k}$ transform in the following way during the transition to the complex axes:

$$
\begin{aligned}
B_{i}^{k} & =\beta_{m}^{n} A_{i}^{m} a_{n}^{k}= \begin{cases}\lambda_{i} & \text { if } i=k \\
0 & \text { if } i \neq k\end{cases} \\
\Gamma_{i}^{k}=\gamma_{m}^{n} A_{i}^{m} a_{n}^{k} & = \begin{cases}\left(\lambda_{i}\right)^{-1} & \text { if } i=k \\
0 & \text { if } i \neq k\end{cases}
\end{aligned}
$$

In terms of the complex axes, one obtains from (11) and $\left(11^{\prime}\right)$ : for polar tensors:

$$
T_{i k l . .}^{p q . .}=\left\{T_{i k l . .}^{p q . .} \lambda_{i} \lambda_{k} \lambda_{l}\left(\lambda_{p}\right)^{-1}\left(\lambda_{q}\right)^{-1} . .\right\}
$$

for axial tensors ${ }^{12}$ :

$$
\lambda_{1} \lambda_{2} \lambda_{3} T_{i k l . .}^{p q . .}=\left\{T_{i k l . .}^{p q . .} \lambda_{i} \lambda_{k} \lambda_{l}\left(\lambda_{p}\right)^{-1}\left(\lambda_{q}\right)^{-1} . .\right\}
$$

where the brackets on the right-hand side indicate that the indices should not be summed up despite the repetition of them.

We have achieved a complete decoupling of the tensor components $T_{i k l . .}^{p q . .}$. Each component is the coefficient in itself of a single homogeneous equation with one unknown and can only be different from zero, independently of the other components, if the product of the $\lambda_{i}$ on both sides of the equation has the same value. If different factors result on both sides, the equation can only be fulfilled if the respective tensor component disappears.

Thus, if there is a tensor of given order, given distribution of covariant and contravariant indices and given polar or axial character, the effect of an element of symmetry on its components can be determined simply by forming the different possible products of $\lambda_{i}$, and comparing for which combinations of axes they satisfy Eqs. $(18)$ or $\left(18^{\prime}\right)$ identically and for which not.

\footnotetext{
12 The product $\lambda_{1} \lambda_{2} \lambda_{3}$ has the value +1 for symmetry operations of the first kind and -1 for those of the second kind. The form given here therefore contains the two forms that the equation can take for the two types of symmetry elements.
} 


\section{Theorems for tensors in symmetric spaces}

We first consider the center of symmetry, $\lambda_{1}=\lambda_{2}=\lambda_{3}=-1$. On the right-hand side of Eqs. (18) and (18'), the tensor component then has the factor +1 or -1 , depending on the order of the tensor is even or odd. On the left-hand side of the equations there is always the factor +1 in the polar case and -1 in the axial case. The equations are fulfilled identically for all tensor components of polar tensors of even order or axial of odd order. In contrast, the factors on the right and left side of the equation are always different for polar tensors of odd order and axial tensors of even order. In these cases, all tensor components disappear. The effect of the center of symmetry can thus be divided into two types of tensors: those which are not influenced by it at all, e.g. dielectric constant, elasticity constants, electrical and thermal conductivity (polar tensors of even order), pyro- and piezomagnetism (axial tensors of odd order) and those which, in the presence of a center of symmetry disappear identically in all components, e.g. pyro- and piezoelectricity (polar tensors odd order) or optical rotation (axial tensors of even order).

As a second element, the reflection plane is examined, $\lambda_{1}=\lambda_{2}=1 ; \lambda_{3}=-1$. The left-hand side of the equation for axial tensors has the factor -1 . One can see that for polar tensors all components can be different of 0 , for which the number of indices 3 is even, whereas for axial tensors only components with an odd number of indices 3 occur. No tensor vanishes completely due to a single reflection plane.

The next element of symmetry we investigate is the axis of rotation, $\lambda_{1}=\mathrm{e}^{\mathrm{i} \varphi}, \lambda_{2}=\mathrm{e}^{-\mathrm{i} \varphi}, \lambda_{3}=+1$. The equations for polar and axial tensors are identical. A distinction between both is therefore not needed in this case. On the left-hand side of all tensor components is the factor +1 , so it is a matter of placing such combinations of $\lambda_{i}$-factors on the right-hand side that the product is +1 as well. Now one sees under all circumstances some combination to achieve this goal: let $o_{1}, o_{2}, o_{3}$ be the number upper indices 1,2 , and 3 , and $u_{1}, u_{2}, u_{3}$ the number of corresponding lower indices. One sees immediately that the product on the right-hand side is +1 if $o_{1}+u_{2}=o_{2}+u_{1}$ holds, because every upper index 1 and each lower 2 represents a multiplication of the tensor component with $\mathrm{e}^{\mathrm{i} \varphi}$, and every upper index 2 or lower 1 represents a multiplication with $\mathrm{e}^{-\mathrm{i} \varphi}$. If there is an equal number of such factors, the product is 1 .

This condition states nothing about the number of indices 3 above or below. Especially no component needs to disappear when the two specified sums are 0 , i.e. where all indices are 3 . An ordinary $n$-fold axis of rotation therefore does not force complete disappearance for any tensor. If the multiplication with the unit roots $\mathrm{e}^{\mathrm{i} \varphi}$ is represented as rotations on the unit circle, the previously mentioned index combinations are characterized by the fact that the same number of rotations around the angle $\varphi$ in clockwise direction as in opposite direction must be executed for this purpose. Naturally, this finally leads to back to the point 1 , regardless of the size of angle $\varphi$, e.g. also for axes of perfect (continuous) cylinder symmetry as they often appear in liquid crystals.

More tensor components, which are unaffected by the above conditions, may be allowed to take nonzero values by the symmetry requirement, namely if a certain value is specified for $\varphi=\frac{2 \pi}{n}$, and the number of factors is large enough to revolve around the unit circle one or more times completely. You can see immediately that at least $n$ steps are necessary, i.e. that the $n$-fold axis of rotation can only be distinguished from cylindrical symmetry if the order of the tensor is at least $n$, because only then there are enough factors available to make new coefficients of tensor components to 1 . This is the reason why, if represented by second-order tensors such as the dielectric constant or the electrical conductivity, one can distinguish systems with at most twofold axes, like the triclinic, monoclinic and rhombic system, but why uniaxial systems behave in the same way regardless of their number of axes, namely cylinder symmetric, and why the cubic system with four trigonal axes appears isotropic for these properties. For piezoelectricity, represented by a third-order constitutive tensor, the trigonal system differs significantly from the tetragonal and hexagonal system, but the two are not different from each other. In the cubic system, the classes whose axes of rotation are at most threefold do not yet behave isotropically; only the two cubic classes with three fourfold axes of rotation show the properties of isotropic bodies.

Finally, the general rotating-reflection axis should be considered: $\lambda_{1}=\mathrm{e}^{\mathrm{i} \varphi}, \lambda_{2}=\mathrm{e}^{-\mathrm{i} \varphi}, \lambda_{3}=-1$. Here again, as with the center and the reflection plane, the factor 1 on the left-hand side stands for polar tensors and the factor -1 for axial tensors. It can therefore be seen that for axes of arbitrarily high fold, i.e. with arbitrarily small $\varphi$, in the case of polar tensors of even order and of axial odd order exactly the same conditions apply as for the rotational axes, but that for axial tensors of even or polar tensors of odd order sufficiently high rotation-reflection axes make all components disappear. The highest rotation-reflection axis of even count that can be distinguished from cylindrical symmetry, has for polar tensors the count $2(n-1)$ and $2 n$ for axial tensors, while the odd-fold rotation-reflection axis can always be composed of a rotational axis and a reflection plane, and therefore obeys the theorems for these two types of symmetry elements simultaneously. 


\section{Composed symmetry classes; backward transformation to real axes}

In the previous paragraph, the actions of the simple symmetry elements have been examined for the tensor components. Among the classes with a compound symmetry, one part can be treated without further ado according to the same method, namely the so-called commutative classes, where all symmetry elements can be simultaneously »transformed to main axes«, i.e. represented by diagonal matrices with complex $\lambda_{1}, \lambda_{2}, \lambda_{3}$. These are all classes of the triclinic, monoclinic and rhombic system, furthermore those uniaxial classes that have, besides an arbitrary rotation or rotation-reflection axis, at most one symmetry center and one horizontal reflection plane. As soon as an axis of higher count than 2 is intersected by another arbitrary axis or a reflection plane normal at a finite angle, it is no longer possible to treat all symmetry elements of the class with a single system of complex axes. Rather, one has to choose the complex axes for the different symmetry elements in different ways, and afterwards transform these axis systems to the same real system and remove all constraints and relations, which are imposed by any of the symmetry elements that are accounted for.

The most convenient way to return from the complex axes to the real is the transition to a Cartesian system of 3 axes of equal length $a_{i}$ and perpendicular to each other. The corresponding transformation is ${ }^{13}$

$$
\begin{aligned}
& a_{1}=\frac{1}{\sqrt{2}}\left(A_{1}+A_{2}\right) \\
& a_{2}=\frac{-\mathrm{i}}{\sqrt{2}}\left(A_{1}-A_{2}\right) \\
& a_{3}=A_{3}
\end{aligned}
$$

$$
\begin{aligned}
& A_{1}=\frac{1}{\sqrt{2}}\left(a_{1}+\mathrm{i} a_{2}\right) \\
& A_{2}=\frac{1}{\sqrt{2}}\left(a_{1}-\mathrm{i} a_{2}\right) \\
& A_{3}=a_{3}
\end{aligned}
$$

If we assume such a Cartesian axis system $a$, the transformation matrices used in (16), (16 $)$ and (17), (17') have the values:

$$
\begin{gathered}
A_{1}^{1}=\frac{1}{\sqrt{2}} ; \quad A_{1}^{2}=\frac{\mathrm{i}}{\sqrt{2}} ; \quad A_{2}^{1}=\frac{1}{\sqrt{2}} ; \quad A_{2}^{2}=\frac{-\mathrm{i}}{\sqrt{2}} ; \quad A_{3}^{3}=1 ; \\
a_{1}^{1}=\frac{1}{\sqrt{2}} ; \quad a_{1}^{2}=\frac{1}{\sqrt{2}} ; \quad a_{2}^{1}=\frac{-\mathrm{i}}{\sqrt{2}} ; \quad a_{2}^{2}=\frac{\mathrm{i}}{\sqrt{2}} ; \quad a_{3}^{3}=1
\end{gathered}
$$

All other $A_{i}^{k}$ and $a_{i}^{k}$ vanish ${ }^{14}$.

It is best to use the system of Eq. (17) by inserting all $T_{I K L . . .}^{P Q .}$, which must disappear on the left-hand side. In this way one will find that each zero in the $T$-tensor corresponds to a linear relationship between the components of the $t$-tensor in real space. Occasionally, however, it is also useful to use the system $\left(17^{\prime}\right)$ and explicitly calculate the real quantities $t_{i k l . .}^{p q .}$ of the non-vanishing components of $T$. But then the linear relations between the components of $t$ are more obscured and not so easy to discover.

The transformation with the help of Eq. (17) has the additional advantage that known symmetry properties of the tensor $t$ can be used. It can be derived from the energy principle that the deformation tensor, which occurs during electrostriction, must not contain any rotation, i.e. that it must be invariant against an exchange of its two indices (at least if one refers to a Cartesian axis system). The same is then true for the corresponding two indices of the third-order tensor of electrostriction and the inverse of piezoelectricity. It can also be deduced from the law of conservation of energy that the tensor of the dielectric constant with respect to Cartesian coordinates must be invariant against interchanging the two indices. Similar relations exist for most physically significant tensors. Therefore such tensor components $t_{i k l . .}^{p q .}$, which must be equal or possibly opposite to each other, will be combined during the transformation. The corresponding relations between the components of $T$ are much more difficult to understand, the easiest way is to equate the linear forms of the right-hand sides of (17) for the respective components of $t$.

\footnotetext{
13 In volume 23 of the Encyclopedia of Physics, ch. 4, by P. P. Ewald, p. 218, where I used the same transformation, I made the mistake of not specifying the intended transformation but the inverse one. Therefore I ask the users of this article to swap the capital and small $a$ in the system of Eq. (15). Please see Ewald [7].

${ }^{14}$ Translator's comment: this basis allows to write rotations around the $a_{3}=A_{3}$ axis conveniently as diagonal matrices. Later on, Hermann refers to the "horizontal axes" as directions perpendicular to $a_{3}=A_{3}$, while $a_{3}=A_{3}$ is called the "vertical axis". This complex basis has the nice property that rotations by $\pi / 2$ around the vertical axis are obtained by multiplying $A_{1}$ and $A_{2}$ by the imaginary unit $i$, which is conveniently summarized with the components while the basis is unaffected; see e.g. Vasil et al. [22].
} 


\section{Example: the elasticity tensor}

To illustrate the calculations described here, a special case shall be examined, namely the elasticity tensor for the different distinguishable groups of crystal classes and for the isotropic body. As long as Hooke's law is fulfilled, elasticity is a linear relationship between a stress tensor and a deformation tensor. The stress is described by a force that acts on a surface element in the body, namely the force $p=p^{i} a_{i}$ which acts on the unit area with the normal $n=n^{i} a_{i}$ given by the tensor $s_{i}^{k}: p^{i}=s_{k}^{i} n^{k}$. If no (microscopic ${ }^{15}$ ) torque is to be exerted, $s_{i}^{k}=s_{k}^{i}$ applies with respect to Cartesian axes. The deformation is given as the displacement $y=y^{i} a_{i}$, which the volume element experiences at the position $x=x^{i} a_{i}$, and which is represented by a deformation tensor $d_{i}^{k}$. In case of a homogeneous distortion the displacement is $y^{i}=d_{k}^{i} x^{k}$.

Here again $d_{k}^{i}=d_{i}^{k}$ is valid, since rotations of matter can be omitted. Hooke's law establishes a linear relationship between these two tensors: $d_{k}^{i}=c_{k n}^{i m} s_{m}^{n}$, where $c_{k n}^{i m}$ is a polar tensor of fourth order. In order for energy conservation to hold there must be an elastic potential $U=\frac{1}{2} d_{k}^{i} s_{i}^{k}=\frac{1}{2} c_{k n}^{i m} s_{i}^{k} s_{m}^{n}$, from which one can calculate the corresponding deformation components by differentiating with respect to the stress components. ${ }^{16}$ This means, however, that the tensor $c_{k n}^{i m}$ must be invariant to the permutation of the left index pair with the right index pair. Thus one has as symmetry conditions for the components of $c: c_{k n}^{i m}=c_{k n}^{i n}=c_{i n}^{k m}=c_{i m}^{k n}=$ $c_{n k}^{m i}=c_{m k}^{n i}=c_{n i}^{m k}=c_{m i}^{n k}$, by which the original $3^{4}=81$ independent components of the tensor $c$ are reduced to 21 independent components.

Of course, these symmetry relations have their simple appearance only as long as one refers to rectangular, Cartesian axes. At the transition to the complex axes they would change significantly. But we can ignore these conditions in the complex axes representation and introduce them only after the transformation into the real space representation.

We now focus on the complex axes (16) and the basic elastic equations $D_{k}^{i},=C_{k n}^{i m} S_{m}^{n}$, whereby we first pretend that there are 81 different components $C_{k n}^{i m}$. Equation (17) becomes for this special case:

$$
C_{k n}^{i m}=C_{k n}^{i m} \lambda_{i} \lambda_{m}\left(\lambda_{k} \lambda_{n}\right)^{-1}
$$

For the center of symmetry, where all $\lambda$ have the value -1 , this equation is always fulfilled identically. Therefore, a center of symmetry does not result in a restriction of the tensor components.

For the horizontal $\mathrm{reflecti}$ on $\mathrm{plan} \mathrm{e},{ }^{17}$ the equation is fulfilled when the number of indices 3 is even; on the other hand, in the presence of this refection plane, the following components must disappear; $C_{13}^{11}, C_{23}^{11}$, $C_{13}^{21}, C_{13}^{12}, C_{31}^{33}$, the same applies for all index permutations of these and when interchanging the indices 1 and 2.

For $n$-fold rotatio o a x e s with $n>4$, only the following components are allowed ${ }^{18}: C_{11}^{11}, C_{12}^{12}, C_{21}^{12}$, $C_{13}^{13}, C_{31}^{31}, C_{31}^{13}, C_{13}^{31}, C_{33}^{12}, C_{12}^{33}, C_{33}^{33}$, the same holds when 1 and 2 are interchanged. For the fourfold axis $C_{22}^{11}$ (and hence $C_{11}^{22}$ ) is also allowed, for the threefold rotation $C_{23}^{11}$ is allowed, the same holds when interchanging 1 and 2 as well as with the 3 at any other place. The twofold axis allows for even more components. This leads to the same restrictions as the horizontal reflection plane. This can also be seen by the fact that an added center of symmetry may not have any influence on the elasticity tensor. Such a center of symmetry would, however, at the same time have an horizontal reflection plane added. For the same reason, a special treatment of the rotation-reflection axis is not necessary, since the corresponding axis of rotation can always be generated from them by adding a center of symmetry.

Now we have to determine the consequences of the vanishing tensor components of $T$ w.r.t. the complex axes for the real components of the tensor $c$ w.r.t. real axes with the help of the transformation (17) and the constants (20). This procedure isss simplified by the fact that all transformation coefficients $A_{i}^{k}$ and $a_{i}^{k}$, where one index is 3 and the other is 1 or 2 , disappear. Thus only those components with indices 3 at the same positions are transformed. For the calculation, we write those as $c_{k n}^{i m}$, which must be identical due to the symmetry relations, among each other.

\footnotetext{
15 Translator's amendment.

16 Translator's comment: Hermann uses for the compliance tetrad the symbols $c$ and $C$ in real and complex space. The common notation nowadays is to use $C$ for the stiffness, i.e. the inverse compliance, and $S$ for the compliance.

17 Translator's comment: "Horizontal" refers to the plane perpendicular to $A_{3}=a_{3}$.

18 Translator's comment: Hermann's point of view is to ask "Which tensor components are allowed by each symmetry transformation." This is contrary to the usual perspective where one presumes all components independent and non-zero, and checks which constraints emerge from symmetry transformations.
} 
For the threefold, fourfold and sixfold axes, the following component vanishes

$$
\begin{array}{r}
C_{12}^{11}=\frac{1}{4}\left(c_{11}^{11}-c_{22}^{22}-c_{12}^{12}+c_{22}^{11}+i c_{21}^{11}+i c_{12}^{22}\right. \\
+c_{22}^{21}-c_{11}^{22}-i c_{12}^{11}-i c_{21}^{22} \\
+c_{12}^{21}-i c_{11}^{12}-i c_{22}^{21} \\
\left.-c_{21}^{12}-i c_{11}^{21}-i c_{22}^{12}\right)
\end{array}
$$

If the usual notation is used, i.e. if index pairs ${ }_{1}^{1}$ are replaced by $1,{ }_{2}^{2}$ by $2,{ }_{3}^{3}$ by $3,{ }_{3}^{2}$ by $4,{ }_{1}^{3}$ by $5,{ }_{2}^{1}$ by 6 , one obtains from $C_{12}^{11}=0$ that the real and imaginary parts must disappear separately, such that two conditions are obtained:

$$
c_{11}=c_{22} \text { and } c_{16}=-c_{26} .
$$

The same restriction is obtained by zeroing all $C$ components with three indices 1 and one 2 or vice versa.

For the threefold and sixfold axis disappears:

$$
\begin{gathered}
C_{22}^{11}=\frac{1}{4}\left(c_{11}^{11}+c_{22}^{22}-c_{12}^{12}+c_{22}^{11}-i c_{21}^{11}+i c_{12}^{22}\right. \\
-c_{21}^{21}-c_{11}^{22}-i c_{11}^{21}+i c_{22}^{12} \\
-c_{12}^{21}-i c_{12}^{11}+i c_{21}^{22} \\
\left.-c_{21}^{12}-i c_{11}^{12}+i c_{22}^{21}\right) \\
\hline=\frac{1}{4}\left(c_{11}+c_{22}-2 c_{12}-4 c_{66}-4 i c_{16}+4 i c_{26}\right) .
\end{gathered}
$$

The two further equations therefore apply to the threefold and sixfold axis:

$$
c_{11}+c_{22}-2 c_{12}-4 c_{66}=0 \text { and } c_{16}=c_{26} .
$$

The same results from $c_{11}^{22}$.

These are the only two types of zeros which appear among the tensor components without index 3 . Thus, one obtains the conditions for these:

For the $\mathrm{t}$ w o f o $1 \mathrm{~d}$ axis: no restrictions, the quantities $c_{11}, c_{22}, c_{12}, c_{16}, c_{26}$, and $c_{66}$ are independent from each other and can take any value.

For the fou r fold axis: only restriction (a): $c_{11}=c_{22} ; c_{16}=-c_{26} ; c_{12}$ and $c_{66}$ are not restricted.

For the threefold and sixfold axes: restrictions (a) and (b) hold simultaneously. Hence, a simple transformation yields: $c_{11}=c_{22} ; c_{16}=c_{26}=0 ; c_{66}=\frac{1}{2}\left(c_{11}-c_{12}\right)$.

The following restrictions can be derived from components with an index 3 :

For the twofold, fourfold and sixfold axes disappears

$$
\begin{array}{r}
C_{23}^{11}=\frac{1}{2 \sqrt{2}}\left(c_{13}^{11}-c_{23}^{21}-c_{23}^{12}+i c_{23}^{22}-i c_{13}^{12}-i c_{23}^{11}\right. \\
\left.-c_{13}^{22}-i c_{13}^{21}\right) \\
=\frac{1}{2 \sqrt{2}}\left[c_{15}-c_{25}-2 c_{46}+i\left(c_{24}-c_{14}-2 c_{56}\right)\right] .
\end{array}
$$

Therefore the following restrictions apply:

$$
c_{15}-c_{25}-2 c_{46}=0 \text { and } c_{24}-c_{14}-2 c_{56}=0 .
$$


For all vertical axes of rotation disappears

$$
\begin{gathered}
C_{13}^{11}=\frac{1}{2 \sqrt{2}}\left(c_{13}^{11}+c_{23}^{21}+c_{23}^{12}-i c_{23}^{22}-i c_{13}^{12}+i c_{23}^{11}\right. \\
\left.-c_{13}^{22}-i c_{13}^{21}\right) \\
=\frac{1}{2 \sqrt{2}}\left[c_{15}+c_{25}-i\left(c_{24}+c_{14}\right)\right] .
\end{gathered}
$$

Therefore it applies here

$$
c_{15}+c_{25}=0 \text { and } c_{14}+c_{24}=0 .
$$

For all vertical ${ }^{19}$ axis of rotation, also disappears

$$
\begin{array}{r}
\begin{array}{r}
C_{13}^{21}=\frac{1}{2 \sqrt{2}}\left(c_{13}^{11}-c_{23}^{21}+\right. \\
+c_{23}^{12}+i c_{23}^{22}-i c_{13}^{12}+i c_{23}^{11} \\
\left.+i c_{13}^{21}\right)
\end{array} \\
=\frac{1}{2 \sqrt{2}}\left[c_{15}-c_{25}+2 c_{46}+i\left(c_{24}-c_{14}+2 c_{56}\right)\right] .
\end{array}
$$

Therefore it applies

$$
c_{15}-c_{25}+2 c_{46}=0 \text { and } c_{24}-c_{14}+2 c_{56}=0
$$

Out of the remaining zeroes of the tensor $C$ with only one index 3 one will always get the same three conditions (c), (d) and (e).

Of conditions (c), (d) and (e), only (d) and (e) apply to the threefold axis. A simple rearrangement gives:

$$
c_{15}=-c_{25}=-c_{46} \text { and } c_{14}=-c_{24}=c_{56} \text {. }
$$

In addition to the $\mathrm{t} w$ ofold, fourfold and sixfold axis it applies (c), i.e. here all components $c_{14}, c_{24}, c_{15}, c_{25}, c_{46}$, and $c_{56}$ have to disappear.

For the threefold, fourfold and sixfold axis, the subsequent term disappears

$$
\begin{aligned}
C_{33}^{11}=\frac{1}{2}\left(c_{33}^{11}-c_{33}^{22}\right. & -i c_{33}^{12} \\
& \left.-i c_{33}^{21}\right)=\frac{1}{2}\left(c_{44}-c_{55}-2 i c_{45}\right) .
\end{aligned}
$$

So it applies

$$
c_{44}=c_{55} \text { and } c_{45}=0 .
$$

For the threefold, fourfold and sixfold axis, the subsequent terms disappears

$$
\begin{aligned}
C_{23}^{13}=\frac{1}{2}\left(c_{23}^{13}-c_{23}^{23}\right. & -i c_{23}^{13} \\
& \left.-i c_{13}^{23}\right)=\frac{1}{2}\left(c_{13}-c_{23}-2 i c_{36}\right) .
\end{aligned}
$$

So it applies

$$
c_{13}=c_{23} \text { and } c_{36}=0 .
$$

For all vertical axes disappears

$$
C_{33}^{13}=\frac{1}{\sqrt{2}}\left(c_{33}^{13}-i c_{33}^{23}\right)=\frac{1}{\sqrt{2}}\left(c_{34}-i c_{35}\right) .
$$

This means

$$
c_{34}=c_{35}=0
$$

All other zero components of $C$ with two or three indices 3 give the same conditions (f), (g) and (h).

This describes all components that the elasticity tensor can have in the presence of a single axis of rotation of any count. It is no difficulty to apply these results in case an axis needs to be considered that is not transformed into the direction of $a_{3}$, but falls in direction $a_{1}$ or $a_{2}$. By including the thereby found conditions, one can easily determine the elasticity tensor for new crystal classes. One finds here exactly the forms that are listed in Voig t (l. c.) 8. 585-587.

19 Translator's comment: "Vertical" refers to the $A_{3}=a_{3}$-axis. 


\section{Summary}

A method was developed to easily derive the influence of symmetry elements on the tensor components for tensorial material constants of any order. The invariance conditions for the individual tensor components are established, not, as is usually the case, for scalar quantities, which must be searched for anew in each case. The resulting complicated determinants can be simplified by suitable choice of axes to such an extent that the result can be found without difficulties.

Institute of Theoretical Physics, Stuttgart, March 1934.

Received March 17, 1934.

Open Access This article is licensed under a Creative Commons Attribution 4.0 International License, which permits use, sharing, adaptation, distribution and reproduction in any medium or format, as long as you give appropriate credit to the original author(s) and the source, provide a link to the Creative Commons licence, and indicate if changes were made. The images or other third party material in this article are included in the article's Creative Commons licence, unless indicated otherwise in a credit line to the material. If material is not included in the article's Creative Commons licence and your intended use is not permitted by statutory regulation or exceeds the permitted use, you will need to obtain permission directly from the copyright holder. To view a copy of this licence, visit http://creativecommons.org/licenses/by/4.0/.

Funding Open Access funding enabled and organized by Projekt DEAL.

\section{A List of symbols}

\begin{tabular}{|c|c|c|}
\hline Our notation & Hermann's notation & Meaning \\
\hline$\pi$ & $\pi$ & Archimedes' constant \\
\hline $\mathrm{e}$ & $e$ & Euler's number \\
\hline $\mathrm{i}$ & $i$ & Imaginary number \\
\hline$\alpha$ & $a$ & Coefficient of thermal expansion \\
\hline$\kappa$ & $c$ & Elastic compressibility \\
\hline$\lambda$ & $\lambda$ & Rotation-reflection axis \\
\hline$\varphi$ & $\varphi$ & Rotation angle \\
\hline$\varepsilon$ & $\varepsilon$ & Absolute permittivity \\
\hline$\sigma$ & $\sigma$ & Electrical conductivity \\
\hline$\theta$ & $T$ & Temperature \\
\hline$c_{p}$ & $c_{p}$ & Specific heat \\
\hline$d$ & $d$ & Lattice plane distance \\
\hline$h_{k}$ & $h_{k}$ & Miller indices \\
\hline$l$ & $l$ & Length \\
\hline$n$ & $n$ & Order of a tensor \\
\hline$o_{i}$ & $o_{i}$ & Number of upper indices $i \in\{1,2,3\}$ \\
\hline$p$ & $p$ & Pressure \\
\hline$s$ & $s$ & Degree of determinant \\
\hline$u_{i}$ & $u_{i}$ & Number of lower indices $i \in\{1,2,3\}$ \\
\hline$Q$ & $u$ & Heat supplied or extracted \\
\hline$U$ & $U$ & Elastic potential \\
\hline$V$ & $V$ & Volume \\
\hline$a_{i}$ & $\mathfrak{a}_{i}$ & Real axes \\
\hline$j$ & j & Current density \\
\hline$n$ & $\mathfrak{n}$ & Normal vector \\
\hline$p$ & $\mathfrak{p}$ & Force vector \\
\hline$u$ & $\mathfrak{y}$ & Displacement vector \\
\hline$x$ & $\mathfrak{x}$ & Position vector \\
\hline$A_{i}$ & $\mathfrak{A}_{i}$ & Complex axes \\
\hline$D$ & $\mathfrak{D}$ & Electric displacement field \\
\hline
\end{tabular}




\begin{tabular}{lll}
\hline Our notation & Hermann's notation & Meaning \\
\hline$E$ & $\mathfrak{E}$ & Electric field/ electric intensivity \\
$\delta_{i}^{k}$ & $\delta_{i}^{k}$ & Kronecker delta \\
$\beta_{i}^{k}$ & $\beta_{i}^{k}$ & Transformation matrix \\
$\gamma_{i}^{k}$ & $\gamma_{i}^{k}$ & Transformation matrix \\
$B_{i}^{k}$ & $B_{i}^{k}$ & Transformation matrix \\
$\Gamma_{i}^{k}$ & $\Gamma_{i}^{k}$ & Transformation matrix \\
$a_{i}^{k}$ & $a_{i}^{k}$ & Unknowns \\
$d_{i}^{k}$ & $d_{i}^{k}$ & Deformation tensor \\
$g_{i k}^{k}$ & $g_{i k}$ & Metric \\
$s_{i}^{k}$ & $s_{i}^{k}$ & Stress tensor \\
$A_{i}^{k}$ & $A_{i}^{k}$ & Unknowns \\
$c_{k n}^{i m}$ & $c_{k n}^{i m}$ & Constitutive tensor (real basis) \\
$C_{k n}^{i m}$ & $C_{k n}^{i m}$ & Constitutive tensor (complex basis) \\
\hline
\end{tabular}

\section{B Mathematica notebook demonstration}

\section{Reduction of the components according to Hermann:}

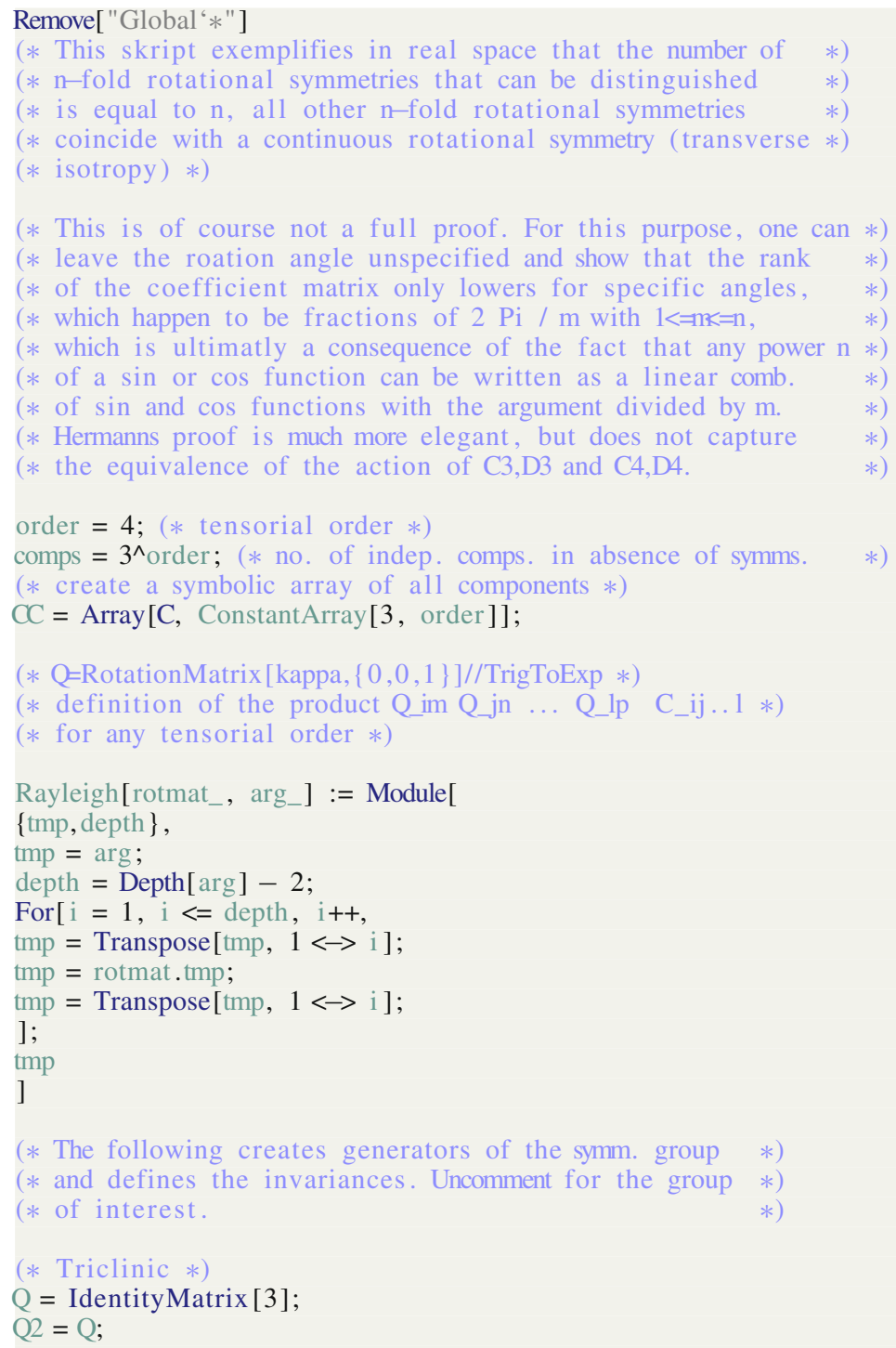




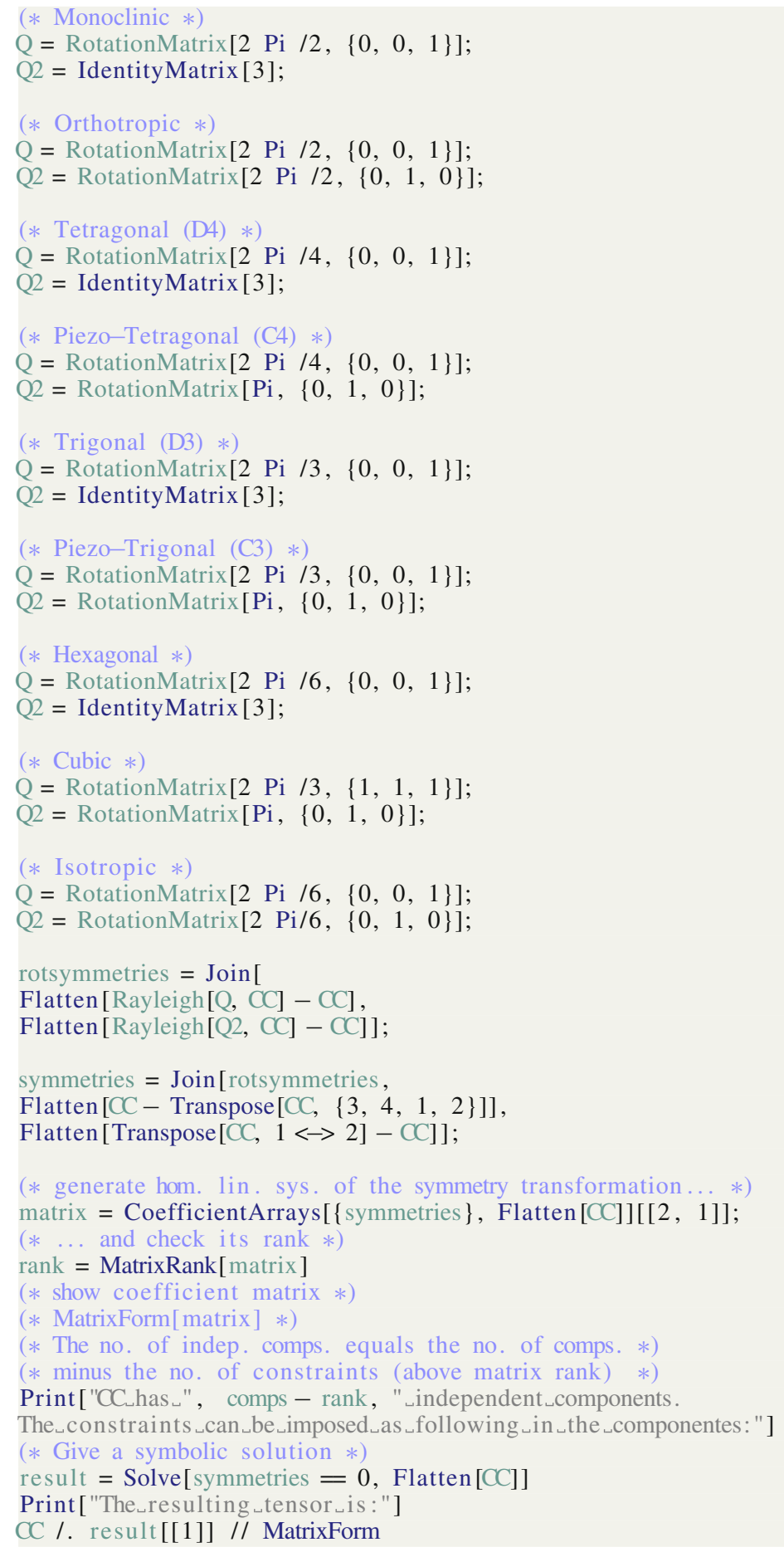


Amendment to show that the reduced stiffnesses from $C 4, D 4$ and $C 3, D 3$ differ only by a rotation:

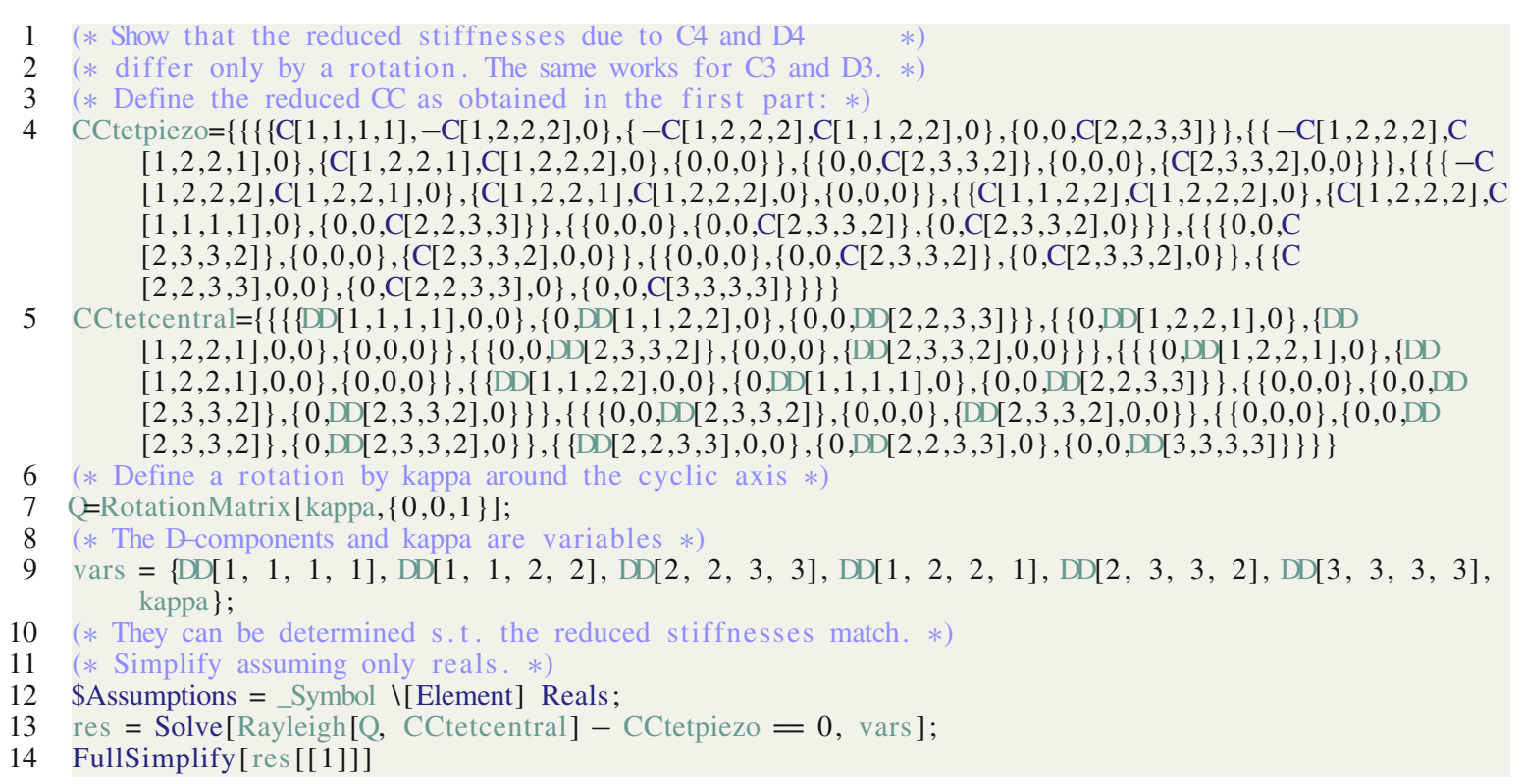

\section{References}

1. Auffray, N., He, Q.C., Le Quang, H.: Complete symmetry classification and compact matrix representations for 3D strain gradient elasticity. Int. J. Solids Struct. 159, 197-210 (2019)

2. Baake, M., Grimm, U.: Aperiodic Order. Aperiodic Order Bd. 1. Cambridge University Press, Cambridge (2013)

3. Chadwick, P., Vianello, M., Cowin, S.C.: A new proof that the number of linear elastic symmetries is eight. J. Mech. Phys. Solids 49(11), 2471-2492 (2001)

4. Cowin, S.C.: On the number of distinct elastic constants associated with certain anisotropic symmetries. Z. Angew. Math. Phys. 46, 210-224 (1995)

5. Curie, P.: Surlasymétrie dans les phénomènes physiques, symétrie d'un champ électrique et d'un champ magnétique. J. Phys. Théor. Appl. 3(1), 393-415 (1894)

6. Eremeyev, V.A., Pietraszkiewicz, W.: Material symmetry group and constitutive equations of micropolar anisotropic elastic solids. Math. Mech. Solids 21(2), 210-221 (2016)

7. Ewald, P.P.: Die Erforschung des Aufbaues der Materie mit Röntgenstrahlen. In: Bothe, W., et al. (eds.) Röntgenstrahlung Ausschliesslich Röntgenoptik: Band XXIII. Zweiter Teil des Handbuchs der Physik, pp. 207-476. Springer, Berlin (1933)

8. Fedorov, F.I.: Theory of Elastic Waves in Crystals. Plenum Press, New York (1968)

9. Forte, S., Vianello, M.: Symmetry classes for elasticity tensors. J. Elast. 43(2), 81-108 (1996)

10. Gurtin, M.: The linear theory of elasticity. In: Vol. VIa/ 2 of Encyclopedia of Physics. Springer, Berlin (1972)

11. Hermann, C.: Über die natürliche optische Aktivität der regulären Kristalle NaClO3 und NaBr O3. Z. Phys. 16(1), 103-134 (1923)

12. Hermann, C.: Die Symmetriegruppen der amorphen und mesomorphen Phasen. Z. Kristallogr. 79(1), 186-221 (1931)

13. Hermann, C.: Tensoren und kristallsymmetrie. Z. Kristallogr. 89(1), 32-48 (1934)

14. Khatkevich, A.G.: The elastic constants of crystals. Sov. Phys. Crystallogr. 6(5), 561-563 (1962)

15. Kowalczyk-Gajewska, K., Ostrowska-Maciejewska, J.: Review on spectral decomposition of Hookes tensor for all symmetry groups of linear elastic material. Eng. Trans. 57(3/4), 145-183 (2009)

16. Lalena, J.N.: From quartz to quasicrystals: probing nature's geometric patterns in crystalline substances. Crystallogr. Rev. 12(2), 125-180 (2006)

17. McCurdy, A.K., Maris, H.J., Elbaum, C.: Anisotropic heat conduction in cubic crystals in the boundary scattering regime. Phys. Rev. B 2(10), 4077-4083 (1970)

18. Neumann, F.E.: Vorlesungen über die Theorie der Elasticität der festen Körper und des Lichtäthers: gehalten an der Universität Königsberg, vol. 5. B.G. Teubner, Leipzig (1885)

19. Nordmann, J., Assmus, M., Altenbach, H.: Visualising elastic anisotropy: theoretical background and computational implementation. Contin. Mech. Thermodyn. 30(4), 689-708 (2018)

20. Renninger, M.: Phys. Blätter. Carl Hermannn 17(12), 584-585 (1961)

21. Ting, T.C.T.: Anisotropic Elasticity: Theory and Applications. Oxford University Press, Oxford (1996)

22. Vasil, G.M., et al.: Tensor calculus in spherical coordinates using Jacobi polynomials. Part-I: Mathematical analysis and derivations. J. Comput. Phys. X 3, 100013 (2019)

23. Voigt, W.: Lehrbuch der Kristallphysik (mit Ausschluss der Kristalloptik). B.G. Teubner, Leipzig (1928)

24. Weyl, H.: Raum, Zeit und Materie: Vorlesungen über Allgemeine Relativitätstheorie, 5th edn. Springer, Berlin (1923) 
25. ХаТКеВИЧ, А. ncyrilicG., “ОБ УПРУГИХ КОНСТАНТАХ КРИСТАЛЛОВ” in: КРИС Т АЛЛОГРАФИЯ 6(5), 700-703

26. Zheng, Q.-S., Zou, W.-N.: Irreducible decompositions of physical tensors of high orders. J. Eng. Math. 37(1-3), 273-288 (2000)

Publisher's Note Springer Nature remains neutral with regard to jurisdictional claims in published maps and institutional affiliations. 